Supporting Information

\title{
Computational Design of 2D Boron-Containing Compounds as Efficient Metal-Free Electrocatalysts toward Nitrogen Reduction Independent on Heteroatom Doping
}

Wei Nongł, Haikuan Liangł, Shihan Qin, Yan Li* and Chengxin Wang

State Key Laboratory of Optoelectronic Materials and Technologies, School of Materials Science and Engineering, Sun Yat-Sen (Zhongshan) University, Guangzhou 510275, People's Republic of China

$\$$ These authors contributed equally to this work

* Corresponding Author. E-mail: liyan266@mail.sysu.edu.cn (Y.L.)

\section{Content}

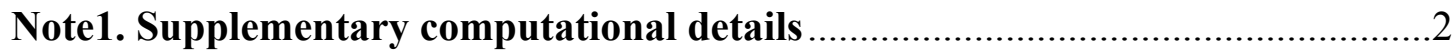

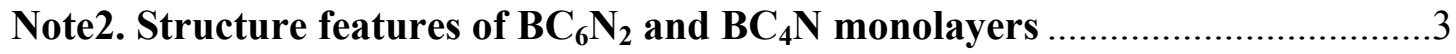

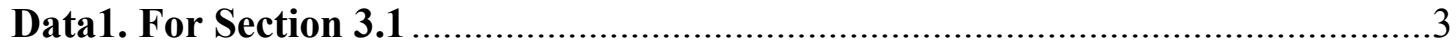

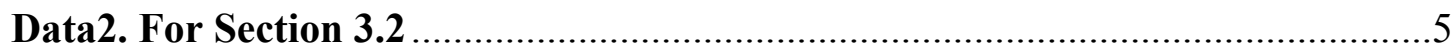

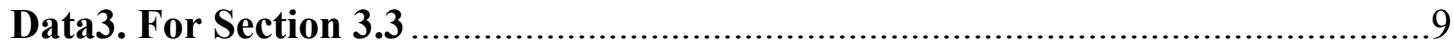

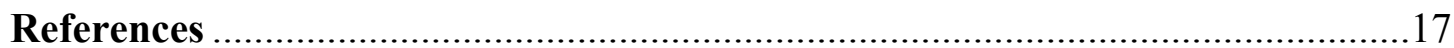




\section{Note1. Supplementary computational details}

The cohesive energy $\left(E_{\mathrm{c}}\right)$ is defined as

$$
E_{\mathrm{c}}=\left(E_{\mathrm{t}}-\Sigma_{i} n_{i} E_{i}\right) / n
$$

Eq. 1

where $E_{\mathrm{t}}$ is the total energy of studied systems, $n_{i}$ and $E_{i}$ are the number of the isolated $i$ atom ( $i=\mathrm{B}, \mathrm{C}$ and $\mathrm{N})$ in unit cell and the corresponding energies, respectively, and $n$ $=\Sigma_{i} n_{i}$ is the total number of atoms. The adsorption energy of $\mathrm{N}_{2}$ molecule and $\mathrm{H}$ atom on substrate is calculated by

$$
\Delta E\left(* \mathrm{~N}_{2}\right)=E\left(* \mathrm{~N}_{2}\right)-E_{\text {sub }}-E\left(\mathrm{~N}_{2}\right)
$$

Eq. 2

and

$$
\Delta E(* \mathrm{H})=E(* \mathrm{H})-E_{\text {sub }}-1 / 2 E\left(\mathrm{H}_{2}\right)
$$

where $E\left(* \mathrm{~N}_{2}\right)$ and $E(* \mathrm{H})$ are the total energies of the substrate with absorbed $\mathrm{N}_{2}$ molecule and $\mathrm{H}$ atom, respectively. $E_{\text {sub }}$ is the total energy of substrate, and $E\left(\mathrm{~N}_{2}\right)$ and $E\left(\mathrm{H}_{2}\right)$ are the energy of $\mathrm{N}_{2}$ and $\mathrm{H}_{2}$ molecule in gas phase. The adsorption sites are denoted as *.

The charge density difference $(\Delta \rho)$ is calculated as

$$
\Delta \rho=\rho\left(\text { sub }-\mathrm{N}_{2}\right)-\rho(\mathrm{sub})-\rho\left(\mathrm{N}_{2}\right)
$$

where $\rho\left(\right.$ sub- $\left.\mathrm{N}_{2}\right)$ and $\rho$ (sub) represent the charge density of the substrates with and without $\mathrm{N}_{2}$ absorbed, respectively, and $\rho\left(\mathrm{N}_{2}\right)$ the charge density of free $\mathrm{N}_{2}$ molecule in the same configuration as the absorbed.

The Gibbs free energy $(G)$ was obtained using the computational hydrogen electrode (CHE) model. ${ }^{1-2}$ At the standard hydrogen electrode (SHE), the chemical potential of $\mathrm{H}^{+} / \mathrm{e}^{-}$pair in aqueous solution is equal to half that of hydrogen gas with pressure of 1 bar. According to CHE model, the free energy change of reaction $(\Delta G)$ for each elementary step of NRR is expressed as

$$
\Delta G=\Delta E-T \Delta S+\Delta \mathrm{ZPE}+\Delta \mathrm{G}_{U}+\Delta G_{\mathrm{pH}}
$$

where $\Delta E$ is the change in total energies of the species involved in each step, $\Delta S$ and $\triangle \mathrm{ZPE}$ are the change in entropy and zero-point energies, respectively. Note that the entropies of gas molecules $\left(\mathrm{N}_{2}, \mathrm{H}_{2}\right.$ and $\left.\mathrm{NH}_{3}\right)$ are obtained from the NIST database ${ }^{3}$, while those of the absorbed intermediates were set to zero. The zero-point energies of both gas molecules and intermediates were calculated using VASP and could be determined as ZPE $=1 / 2 \Sigma_{i} h v_{i}$, here $h$ and $v_{i}$ are Planck constant and vibrational frequencies, respectively. $T$ is the temperature and was set as $298.15 \mathrm{~K}$ here. $\Delta G_{U}=$ $n \mathrm{e} U$ is the free energy contributed by electrode potential, here $n$ e and $U$ are the number of electrons transferred and the applied electrode potential, respectively. $\Delta G_{\mathrm{pH}}=k_{\mathrm{B}} T \times$ $\ln 10 \times \mathrm{pH}$ is the correction of $\mathrm{pH}$, here $k_{\mathrm{B}}$ is the Boltzmann constant, and $\mathrm{pH}=0$ was considered in this work. The limiting potential $\left(U_{\mathrm{L}}\right)$ of the whole process is used to estimate the performance of NRR catalysts, which is determined by the potentialdetermining step (PDS) that has the maximum $\Delta G$ values $\left(\Delta G_{\mathrm{PDS}}\right)$, and can be calculated by

$$
U_{\mathrm{L}}=-\Delta G_{\mathrm{PDS}} / e
$$

If $\Delta G_{\mathrm{PDS}}$ is less than $0.15 \mathrm{eV}, U_{\mathrm{L}}$ is set to the equilibrium potential of NRR $(-0.15 \mathrm{~V}){ }^{4}$ All of the potential values are referenced to SHE. The thermodynamic quantities of $\mathrm{N}_{2}$, $\mathrm{H}_{2}, \mathrm{NH}_{3}$ species in the gas phase are listed in Table S3. 


\section{Note2. Structure features of $\mathrm{BC}_{6} \mathrm{~N}_{2}$ and $\mathrm{BC}_{4} \mathbf{N}$ monolayers}

There are $24 \mathrm{C}, 8 \mathrm{~N}$ and $4 \mathrm{~B}$ atoms in a $\mathrm{BC}_{6} \mathrm{~N}_{2}$ unit cell, while the $\mathrm{BC}_{4} \mathrm{~N}$ unit cell contains $12 \mathrm{C}, 3 \mathrm{~N}$ and $3 \mathrm{~B}$ atoms. We can find in both systems holes and $\mathrm{B}$ atoms are distributed uniformly in the basal plane. The $\mathrm{BC}_{6} \mathrm{~N}_{2}$ monolayer is composed of three kinds of six-membered ring, i.e., $\mathrm{C}_{6}, \mathrm{C}_{4} \mathrm{~N}_{2}$ and $\mathrm{C}_{4} \mathrm{~B}_{2}$ rings. Each $\mathrm{C}_{6}$ ring is edge-sharing with two $\mathrm{C}_{4} \mathrm{~N}_{2}$ rings and $\mathrm{C}_{4} \mathrm{~B}_{2}$ ring. The hole possesses two $\mathrm{B}$ atoms, one faces another. The $\mathrm{BC}_{4} \mathrm{~N}$ monolayer consists of two six-membered rings, $\mathrm{C}_{6}$ and $\mathrm{BC}_{4} \mathrm{~N}$ rings, and every $\mathrm{C}_{6}$ ring is surrounded by three $\mathrm{BC}_{4} \mathrm{~N}$ rings. The average length of $\mathrm{C}-\mathrm{C}$ bonds in $\mathrm{BC}_{4} \mathrm{~N}$ is $1.45 \AA$, while $\mathrm{C}-\mathrm{N}$ bonds and $\mathrm{B}-\mathrm{C}$ bonds are $1.35 \AA$ and $1.44 \AA$, respectively. The bond lengths in $\mathrm{BC}_{6} \mathrm{~N}_{2}$ and $\mathrm{BC}_{4} \mathrm{~N}$ are shown in Figure S3. The average length of C-C bonds $(1.44 \AA)$ is similar to that in graphene ${ }^{5}(1.42 \AA)$, and the length of $\mathrm{B}-\mathrm{C}$ bonds are 1.46 and $1.44 \AA$ for $\mathrm{BC}_{6} \mathrm{~N}_{2}$ and $\mathrm{BC}_{4} \mathrm{~N}$, respectively, while the $\mathrm{C}-\mathrm{N}$ bond are shorter, within the scope of $1.34 \sim 1.35 \AA$.

\section{Data1. For Section 3.1}

Table S1. Several B containing holey $2 \mathrm{D}$ compounds $\left(\mathrm{B}_{x} \mathrm{C}_{2} \mathrm{~N}_{1-x}\right)$ derived from $\mathrm{C}_{2} \mathrm{~N}$.

\begin{tabular}{ccccc}
\hline \hline Formula & B content $(x)$ & $E_{\mathrm{c}}(\mathrm{eV})$ & Space group & Stable? \\
\hline $\mathrm{C}_{2} \mathrm{~N}$ & 0 & -6.77 & $P 6 / \mathrm{mmm}$ & $\sqrt{ }$ \\
$\mathrm{BC}_{6} \mathrm{~N}_{2}$ & $1 / 3$ & -6.73 & $C m m m$ & $\sqrt{ }$ \\
$\mathrm{BC}_{4} \mathrm{~N}$ & $1 / 2$ & -6.73 & $P-62 m$ & $\sqrt{ }$ \\
$\mathrm{B}_{2} \mathrm{C}_{6} \mathrm{~N}$ & $2 / 3$ & -6.59 & $C m m m$ & $\times$ \\
$\mathrm{C}_{2} \mathrm{~B}$ & 1 & -6.50 & $P 6 / \mathrm{mmm}$ & $\times$ \\
\hline \hline
\end{tabular}
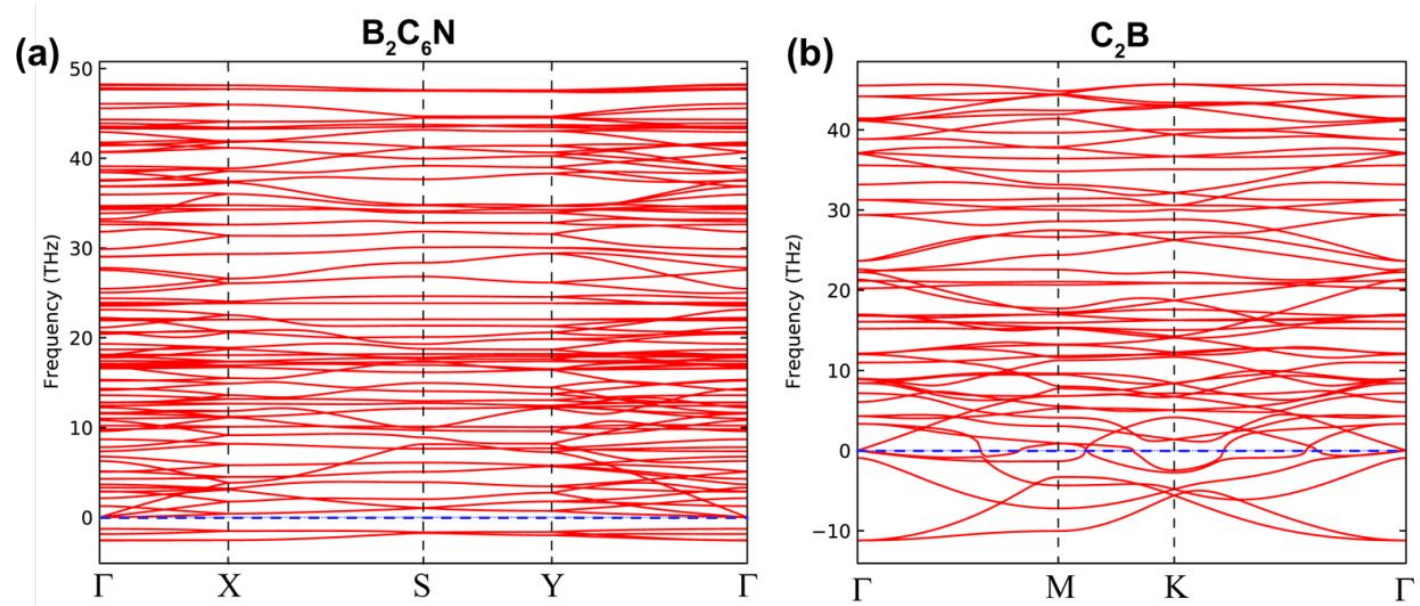

Figure S1. Phonon spectrums of (a) $\mathrm{B}_{2} \mathrm{C}_{6} \mathrm{~N}$ and (b) $\mathrm{C}_{2} \mathrm{~B}$ monolayers. 

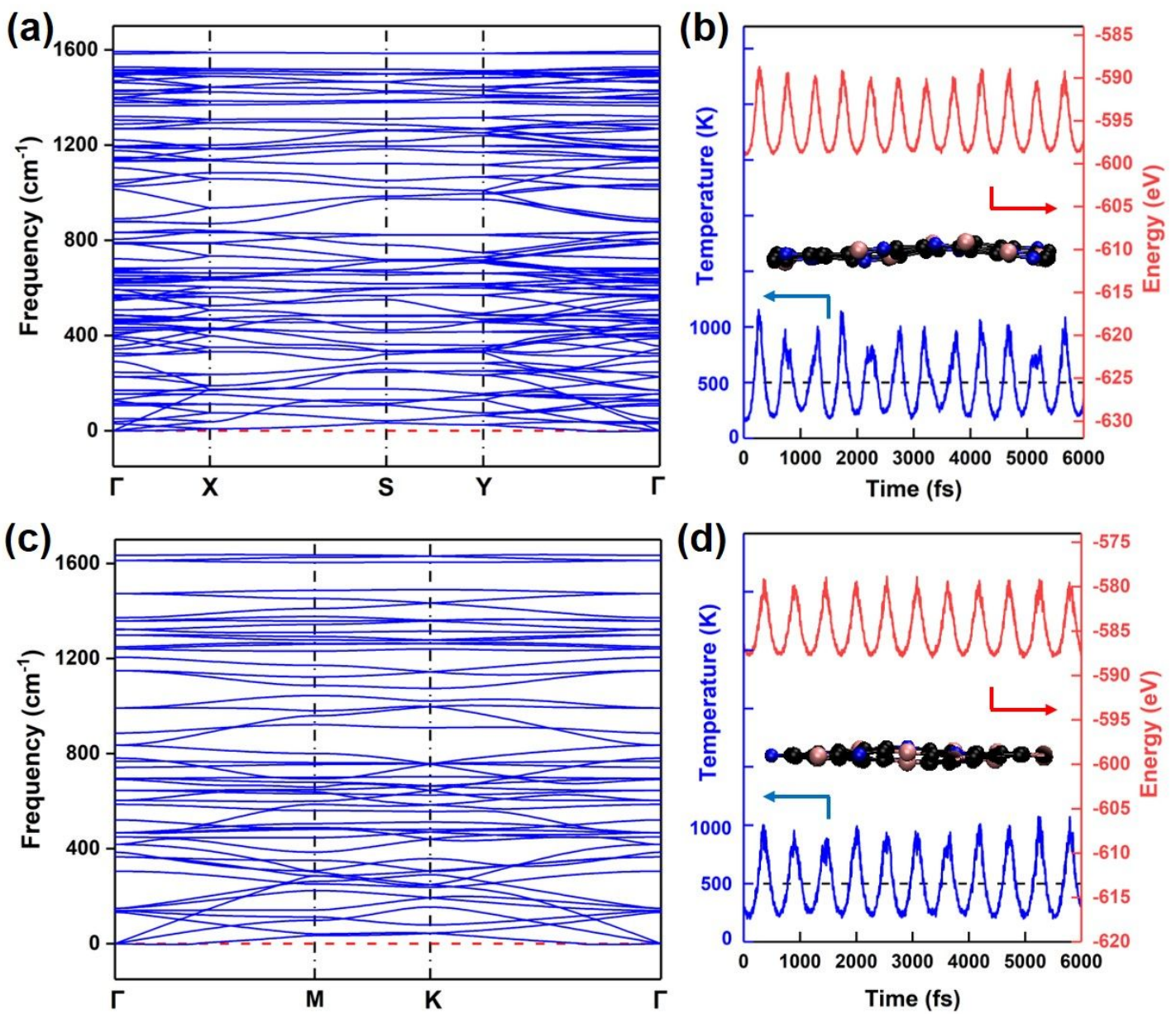

Figure S2. Phonon spectrums and AIMD simulations for $\mathrm{BC}_{6} \mathrm{~N}_{2}(\mathrm{a}, \mathrm{b})$ and $\mathrm{BC}_{4} \mathrm{~N}(\mathrm{c}$, d). The lateral views of monolayers at the end of AIMD simulations are illustrated in the insets $(b, d)$.

Table S2. Structural information for $\mathrm{BC}_{6} \mathrm{~N}_{2}$ and $\mathrm{BC}_{4} \mathrm{~N}$ monolayers.

\begin{tabular}{llll}
\hline \hline Formula & $\begin{array}{l}\text { Space } \\
\text { group }\end{array}$ & Lattice parameters & $\begin{array}{c}\text { Wyckoff position (fractional) } \\
\text { Atom(letter), } x, y, z\end{array}$ \\
\hline $\mathrm{BC}_{6} \mathrm{~N}_{2}$ & $C m m m$ & $a=15.21526 \AA$ & $\mathrm{B}(4 \mathrm{~g}), 0.00000,0.64580,0.50000$ \\
& & $b=8.35423 \AA$ & $\mathrm{C}(8 \mathrm{p}), 0.74559,0.58782,0.50000$ \\
& & $c=20.00000 \AA$ & $\mathrm{C}(8 \mathrm{p}), 0.82763,0.32770,0.50000$ \\
& $\gamma=90.0000^{\circ}$ & $\mathrm{C}(8 \mathrm{p}), 0.90969,0.58764,0.50000$ \\
& & $\mathrm{~N}(8 \mathrm{p}), 0.16921,0.83336,0.50000$ \\
\hline $\mathrm{BC}_{4} \mathrm{~N}$ & $P-62 m$ & $a=8.67697 \AA$ & $\mathrm{B}(3 \mathrm{~g}), 0.65511,0.65511,0.50000$ \\
& & $b=8.67697 \AA$ & $\mathrm{C}(6 \mathrm{k}), 0.64887,0.15657,0.50000$ \\
& & $c=20.00000 \AA$ & $\mathrm{C}(6 \mathrm{k}), 0.82121,0.31534,0.50000$ \\
& $\gamma=120.0000^{\circ}$ & $\mathrm{N}(3 \mathrm{~g}), 0.35286,0.35286,0.50000$ \\
\hline \hline
\end{tabular}



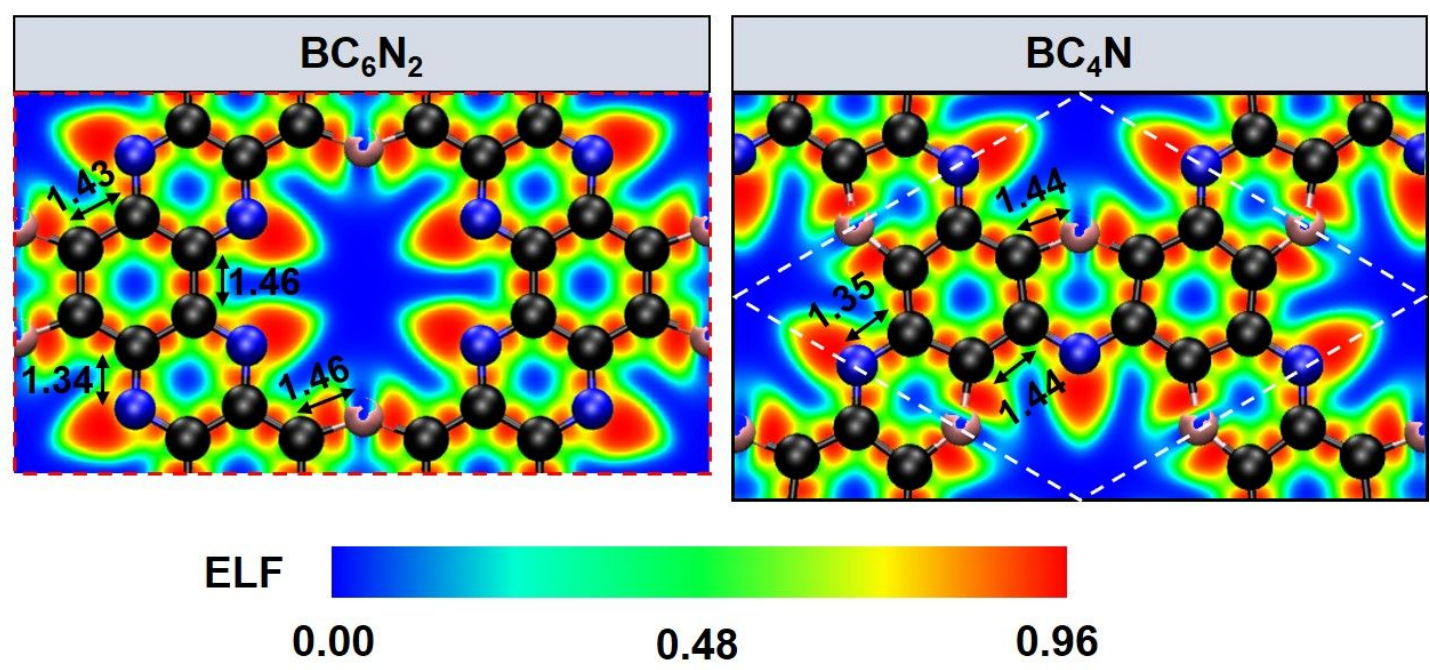

Figure S3. The ELF maps of $2 \mathrm{D} \mathrm{BC}_{6} \mathrm{~N}_{2}$ and $\mathrm{BC}_{4} \mathrm{~N}$ monolayer. Bond lengths are in $\AA$.

\section{Data2. For Section 3.2}
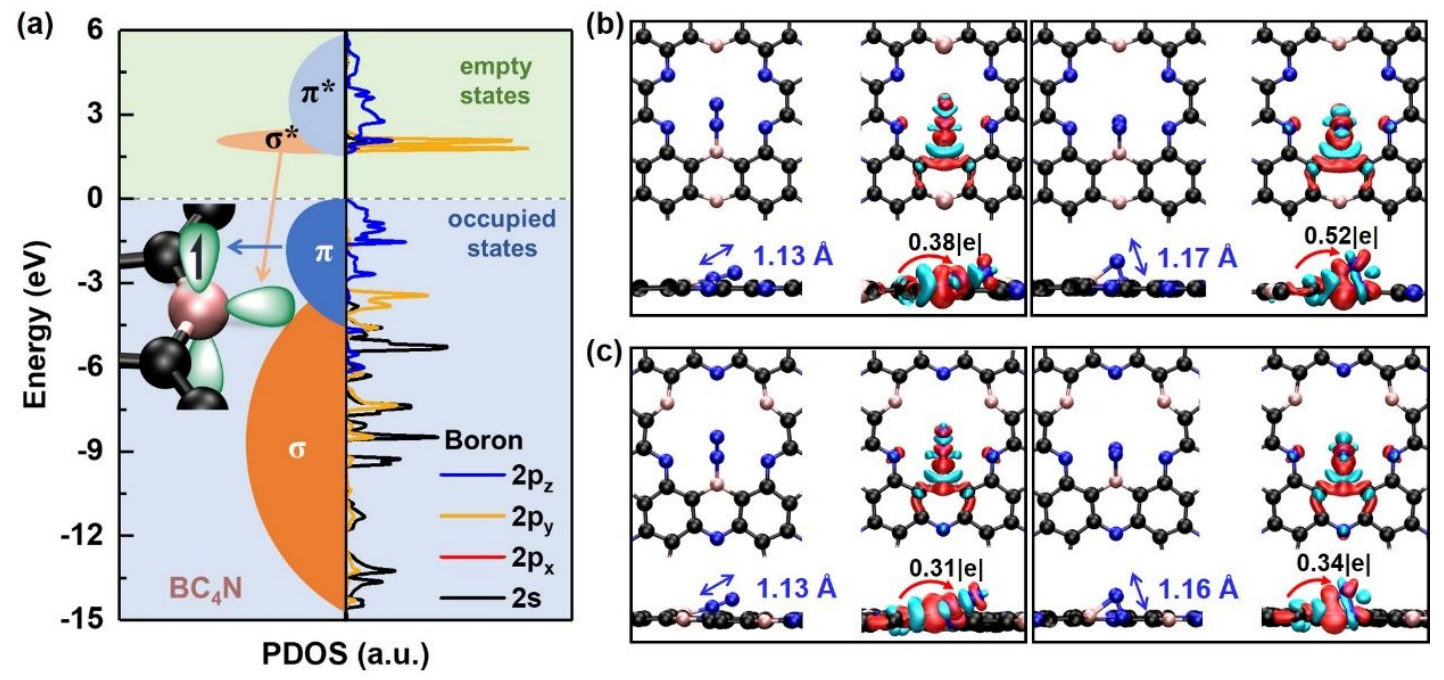

Figure S4. (a) The orbital projected DOS of B in $\mathrm{BC}_{4} \mathrm{~N}$. Note that below the $E_{\mathrm{f}}, \mathrm{B} 2 p_{x}$ is masked by $2 p_{y} .(\mathrm{b}, \mathrm{c})$ The top and side views of structures and corresponding charge density difference of end-on and side-on $\mathrm{N}_{2}$ on (b) $\mathrm{BC}_{6} \mathrm{~N}_{2}$ and (c) $\mathrm{BC}_{4} \mathrm{~N}$. The red and cyan zones represent charge accumulation and depletion, respectively. The bond length of $\mathrm{N}-\mathrm{N}$ of absorbed $\mathrm{N}_{2}$ is illustrated.
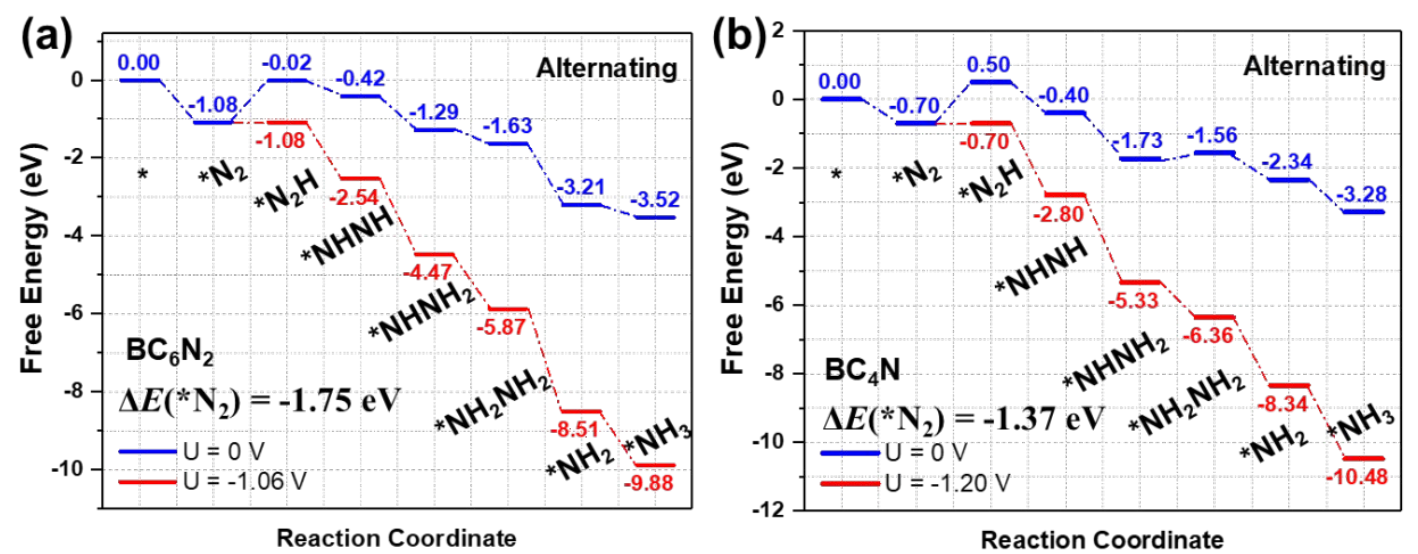

Figure S5. Free energy profiles for $\mathrm{N}_{2}$ reduction on (a) $\mathrm{BC}_{6} \mathrm{~N}_{2}$ and (b) $\mathrm{BC}_{4} \mathrm{~N}$ through alternating mechanisms. 


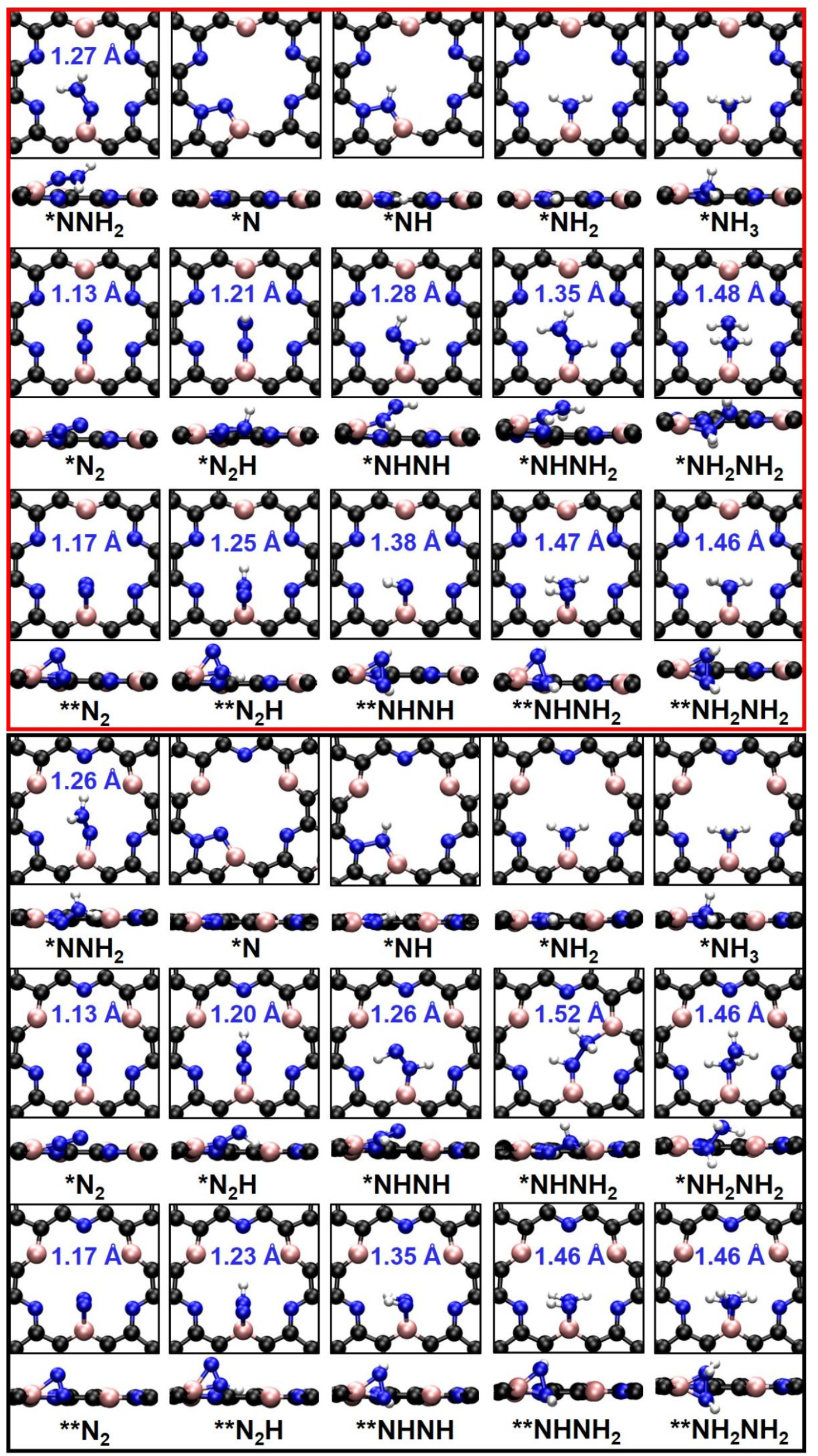

Figure S6. Top and side views of intermediates on (upper) $\mathrm{BC}_{6} \mathrm{~N}_{2}$ and (lower) $\mathrm{BC}_{4} \mathrm{~N}$. The bond length of $\mathrm{N}-\mathrm{N}$ before broken is illustrated. 
(a)
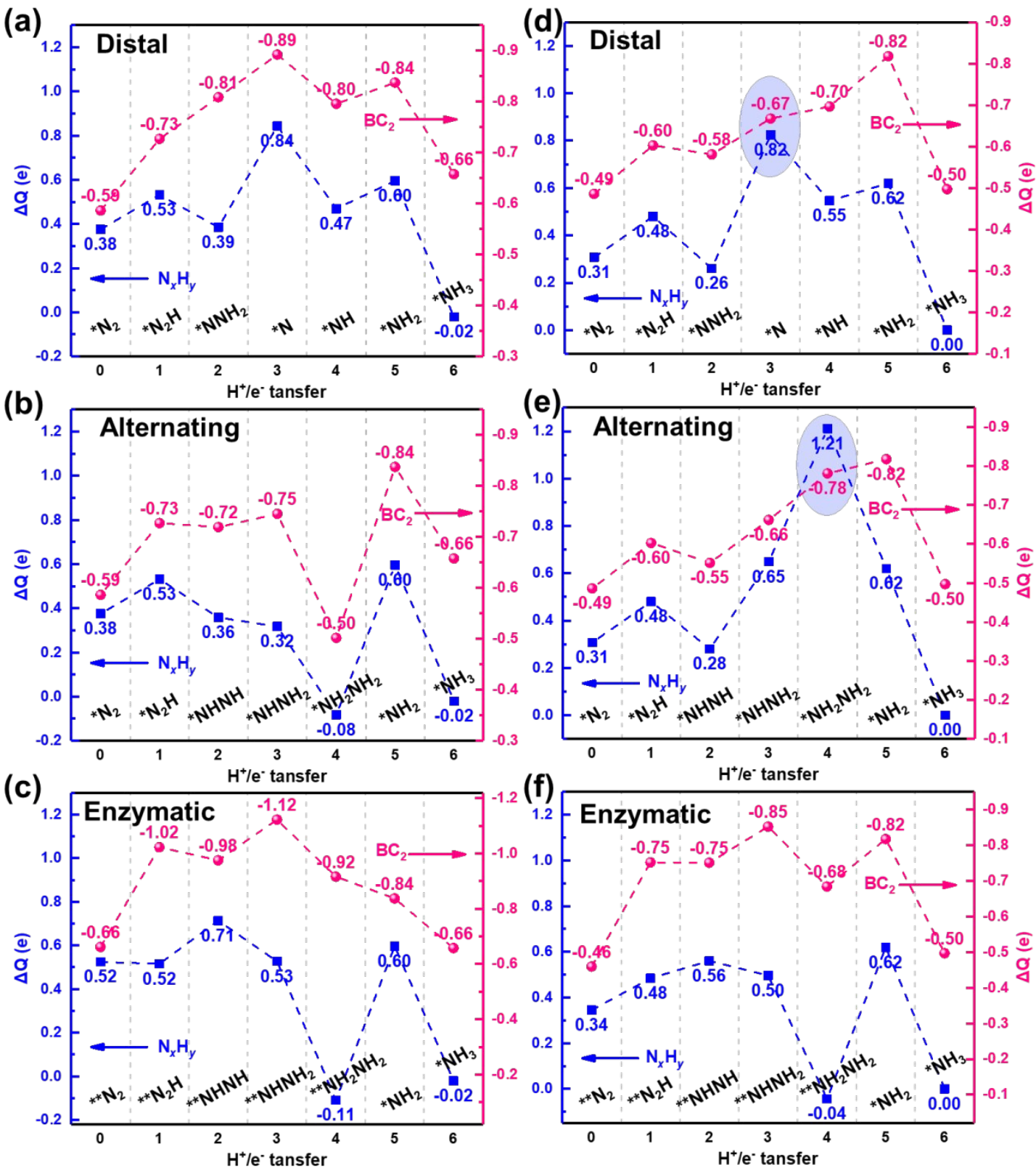

Figure S7. Charge variations of $\mathrm{BC}_{2}$ unit (moiety1) and $\mathrm{N}_{x} \mathrm{H}_{y}$ absorbates (moiety2) during NRR process on $\mathrm{BC}_{6} \mathrm{~N}_{2}(\mathrm{a}, \mathrm{b}, \mathrm{c})$ and $\mathrm{BC}_{4} \mathrm{~N}(\mathrm{~d}, \mathrm{e}, \mathrm{f})$ via distal, alternating and enzymatic mechanisms. Note that $\Delta Q($ moiety 3$)=-(\Delta Q($ moiety 1$)+\Delta Q($ moiety 2$))$.

Table S3 The calculated total energies $\left(E_{\mathrm{t}}\right)$ and thermodynamic quantities of gas phase $\mathrm{N}_{2}, \mathrm{H}_{2}$ and $\mathrm{NH}_{3}$ species. Here, $\mathrm{T}=298.15 \mathrm{~K}$, and experimental entropies are adopted from NIST Standard Reference Database: http:/cccbdb.nist.gov/.

\begin{tabular}{cccccc}
\hline \hline Species & $E_{\mathrm{t}}(\mathrm{eV})$ & $S_{\text {exp }}\left(\mathrm{J} \cdot \mathrm{mol}^{-1} \cdot \mathrm{K}^{-1}\right)$ & $\mathrm{TS}(\mathrm{eV})$ & $\mathrm{ZPE}(\mathrm{eV})$ & $G(\mathrm{eV})$ \\
\hline $\mathrm{N}_{2}$ & -16.63 & 191.61 & 0.59 & 0.16 & -17.07 \\
$\mathrm{H}_{2}$ & -6.74 & 130.68 & 0.40 & 0.29 & -6.85 \\
$\mathrm{NH}_{3}$ & -19.54 & 192.77 & 0.60 & 0.93 & -19.21 \\
\hline \hline
\end{tabular}


Table S4. The calculated thermodynamic quantities of intermediates in NRR process on $\mathrm{BC}_{6} \mathrm{~N}_{2}$ and $\mathrm{BC}_{4} \mathrm{~N}$. Here, $E *$ represents the total energy of substrate.

\begin{tabular}{|c|c|c|c|}
\hline \multicolumn{4}{|c|}{$\mathrm{BC}_{6} \mathrm{~N}_{2}$} \\
\hline Intermediates & $E_{\mathrm{t}}-E_{*}(\mathrm{eV})$ & ZPE (eV) & $G(\mathrm{eV})$ \\
\hline$* \mathrm{~N}_{2}$ & -18.35 & 0.24 & -18.11 \\
\hline$* * \mathrm{~N}_{2}$ & -17.24 & 0.21 & -17.03 \\
\hline$* \mathrm{~N}_{2} \mathrm{H}$ & -21.43 & 0.51 & -20.92 \\
\hline$* * \mathrm{~N}_{2} \mathrm{H}$ & -20.50 & 0.52 & -19.98 \\
\hline$* \mathrm{NNH}_{2}$ & -25.50 & 0.86 & -24.64 \\
\hline *NHNH & -25.19 & 0.86 & -24.33 \\
\hline **NHNH & -24.56 & 0.85 & -23.71 \\
\hline$* \mathrm{~N}$ & -8.61 & 0.12 & -8.50 \\
\hline$* \mathrm{NHNH}_{2}$ & -29.80 & 1.18 & -28.62 \\
\hline$* * \mathrm{NHNH}_{2}$ & -29.61 & 1.19 & -28.42 \\
\hline *NH & -14.07 & 0.45 & -13.62 \\
\hline$* \mathrm{NH}_{2} \mathrm{NH}_{2}$ & -33.90 & 1.51 & -32.39 \\
\hline$* * \mathrm{NH}_{2} \mathrm{NH}_{2}$ & -33.43 & 1.51 & -31.92 \\
\hline$* \mathrm{NH}_{2}$ & -19.09 & 0.76 & -18.33 \\
\hline$* \mathrm{NH}_{3}$ & -23.20 & 1.07 & -22.13 \\
\hline \multicolumn{4}{|c|}{$\mathrm{BC}_{4} \mathrm{~N}$} \\
\hline State & $E_{\mathrm{t}}-E_{*}(\mathrm{eV})$ & ZPE (eV) & $G(\mathrm{eV})$ \\
\hline$* \mathrm{~N}_{2}$ & -18.00 & 0.23 & -17.77 \\
\hline$* * \mathrm{~N}_{2}$ & -16.75 & 0.19 & -16.56 \\
\hline$* \mathrm{~N}_{2} \mathrm{H}$ & -20.50 & 0.50 & -20.00 \\
\hline$* * \mathrm{~N}_{2} \mathrm{H}$ & -19.62 & 0.50 & -19.11 \\
\hline$* \mathrm{NNH}_{2}$ & -24.79 & 0.86 & -23.93 \\
\hline *NHNH & -25.22 & 0.90 & -24.32 \\
\hline **NHNH & -23.83 & 0.84 & -22.99 \\
\hline$* \mathrm{~N}$ & -7.93 & 0.11 & -7.82 \\
\hline$* \mathrm{NHNH}_{2}$ & -30.28 & 1.21 & -29.07 \\
\hline$* * \mathrm{NHNH}_{2}$ & -28.67 & 1.18 & -27.49 \\
\hline$* \mathrm{NH}$ & -13.06 & 0.43 & -12.63 \\
\hline$* \mathrm{NH}_{2} \mathrm{NH}_{2}$ & -33.81 & 1.48 & -32.33 \\
\hline$* * \mathrm{NH}_{2} \mathrm{NH}_{2}$ & -33.13 & 1.49 & -31.64 \\
\hline$* \mathrm{NH}_{2}$ & -18.07 & 0.74 & -17.33 \\
\hline$* \mathrm{NH}_{3}$ & -22.75 & 1.06 & -21.69 \\
\hline
\end{tabular}




\section{Data3. For Section 3.3}
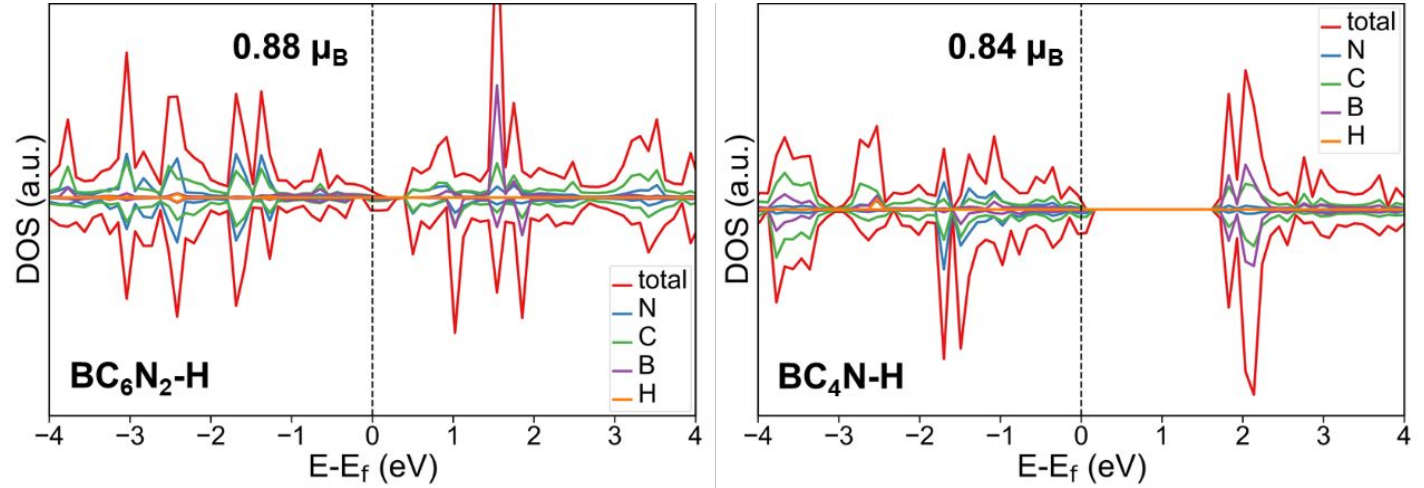

Figure S8. The spin-polarized elemental projected DOS (ePDOS) of $\mathrm{BC}_{6} \mathrm{~N}_{2}-\mathrm{H}$ and $\mathrm{BC}_{4} \mathrm{~N}-\mathrm{H}$. The magnetism is in Bohr momentum $\left(\mu_{\mathrm{B}}\right)$ per cell.
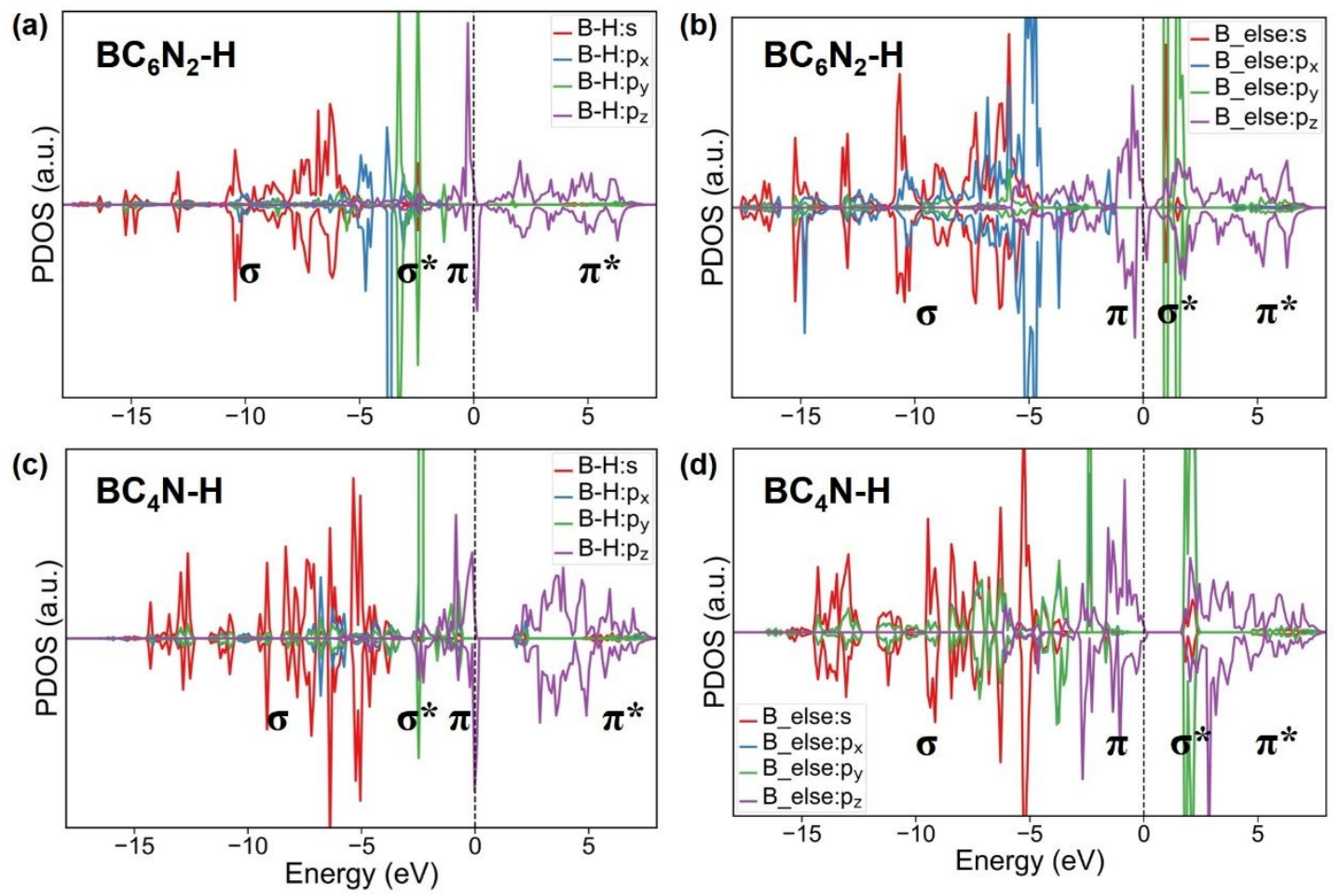

Figure S9. Orbital projected DOS of (a, c) B-H atom (pre-absorbed with $\mathrm{H}$ ) and (b, d) other $\mathrm{B}$ atoms for $\mathrm{BC}_{6} \mathrm{~N}_{2}-\mathrm{H}$ and $\mathrm{BC}_{4} \mathrm{~N}-\mathrm{H}$ monolayers.

Table S5 The adsorption energies of hydrogen. $\Delta E\left({ }^{*} \mathrm{H}\right)$ and $\Delta G\left({ }^{*} \mathrm{H}\right)$ are in $\mathrm{eV}$.

\begin{tabular}{|c|c|c|c|c|c|}
\hline \multicolumn{3}{|c|}{$\mathrm{BC}_{6} \mathrm{~N}_{2}$} & \multicolumn{3}{|c|}{$\mathrm{BC}_{4} \mathrm{~N}$} \\
\hline \multirow[t]{6}{*}{ Site } & \multicolumn{2}{|c|}{$\Delta E(* \mathrm{H}) / \Delta \boldsymbol{G}\left({ }^{*} \mathbf{H}\right)$} & \multirow[t]{2}{*}{ Site } & \multicolumn{2}{|c|}{$\Delta E(* \mathrm{H}) / \Delta \boldsymbol{G}(* \mathbf{H})$} \\
\hline & B & $-1.54 /-1.23$ & & $B$ & $-0.79 /-\mathbf{0 . 4 8}$ \\
\hline & $\mathrm{N}$ & $-0.54 /-\mathbf{0 . 1 6}$ & & $N$ & $-0.09 / \mathbf{0 . 2 9}$ \\
\hline & $\mathrm{C}$ & $0.69 / \mathbf{1 . 0 5}$ & & & $1.13 / 1.48$ \\
\hline & $\mathrm{C}$ & $0.93 / 1.29$ & & & $1.46 / 1.82$ \\
\hline & $\mathrm{C}$ & $1.08 / \mathbf{1 . 4 5}$ & & & - \\
\hline
\end{tabular}


Table S6. The $\mathrm{N}_{2}$ adsorption energies on $\mathrm{BC}_{6} \mathrm{~N}_{2}-\mathrm{H}$ and $\mathrm{BC}_{4} \mathrm{~N}-\mathrm{H}$ at various nonequivalent active sites. $\Delta E\left(* \mathrm{~N}_{2}\right)$ is in $\mathrm{eV}$.

\begin{tabular}{ccc|ccc}
\hline \hline $\mathrm{BC}_{6} \mathrm{~N}_{2}-\mathrm{H}$ & Configuration & $\Delta E\left(* \mathrm{~N}_{2}\right)$ & $\mathrm{BC}_{4} \mathrm{~N}-\mathrm{H}$ & Configuration & $\Delta E\left(* \mathrm{~N}_{2}\right)$ \\
\hline \multirow{2}{*}{ pristine } & end-on & -1.75 & pristine & end-on & -1.37 \\
& side-on & -0.61 & & side-on & -0.11 \\
\hline \multirow{2}{*}{$\mathrm{S} 1$} & end-on & -1.71 & \multirow{2}{*}{$\mathrm{S} 1$} & end-on & $-\mathbf{1 . 4 1}$ \\
& side-on & $\mathbf{- 0 . 1 6}$ & & side-on & -0.11 \\
\hline \multirow{2}{*}{$\mathrm{S} 2$} & end-on & $\mathbf{- 1 . 3 8}$ & \multirow{2}{*}{$\mathrm{S} 2$} & end-on & $\mathbf{- 1 . 3 1}$ \\
& side-on & $\mathbf{- 0 . 5 9}$ & & side-on & $-\mathbf{0 . 0 7}$ \\
\hline \multirow{2}{*}{$\mathrm{S} 3$} & end-on & -1.72 & \multirow{2}{*}{$\mathrm{S} 3$} & end-on & -1.37 \\
& side-on & -0.58 & & side-on & -0.11 \\
\hline \multirow{2}{*}{$\mathrm{S} 4$} & end-on & -1.72 & \multirow{2}{*}{$\mathrm{S} 4$} & end-on & -1.37 \\
& side-on & -0.54 & & side-on & $-\mathbf{0 . 1 2}$ \\
\hline \multirow{2}{*}{$\mathrm{S} 5$} & end-on & -1.73 & \multirow{2}{*}{$\mathrm{S} 5$} & end-on & -1.35 \\
& side-on & -0.53 & & side-on & -0.10 \\
\hline \multirow{2}{*}{$\mathrm{S} 6$} & end-on & $-\mathbf{1 . 7 5}$ & \multirow{2}{*}{$\mathrm{S} 6$} & end-on & -1.37 \\
& side-on & -0.58 & & side-on & -0.11 \\
\hline \multirow{2}{*}{$\mathrm{S} 7$} & end-on & -1.73 & & & \\
\hline \hline
\end{tabular}

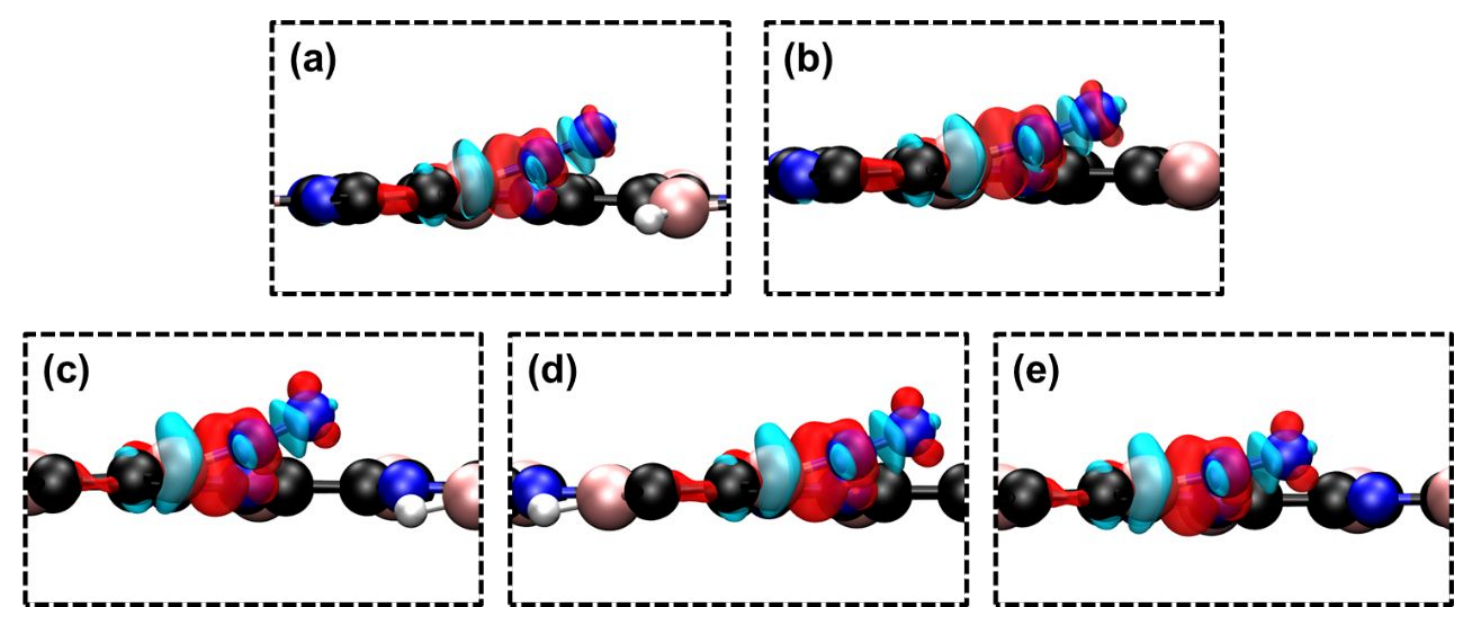

Figure S10. Side views of the charge density difference of end-on adsorbed $\mathrm{N}_{2}$ at (a, b) $\mathrm{BC}_{4} \mathrm{~N}-\mathrm{H}: \mathrm{S} 1 / \mathrm{S} 5$ and $(\mathrm{c}, \mathrm{d}, \mathrm{e}) \mathrm{BC}_{6} \mathrm{~N}_{2}-\mathrm{H}: \mathrm{S} 1 / \mathrm{S} 2 / \mathrm{S} 5$. The red and cyan zones represent charge accumulation and depletion, respectively. The isosurface value is set to 0.03 $\mathrm{e} / \AA^{3}$. 
(a) Distal

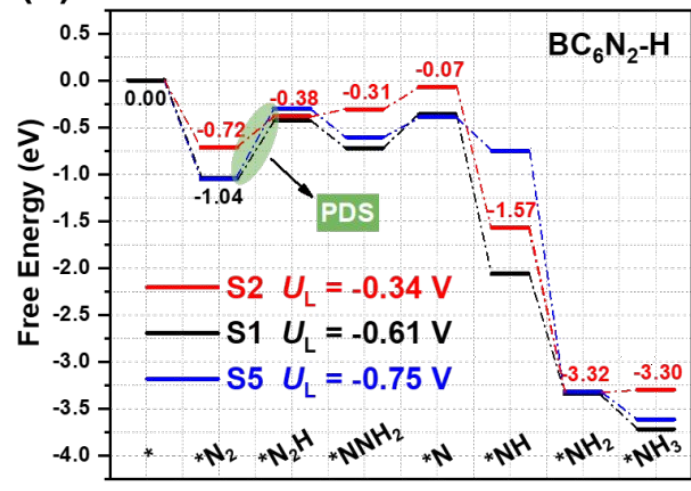

(b) Distal

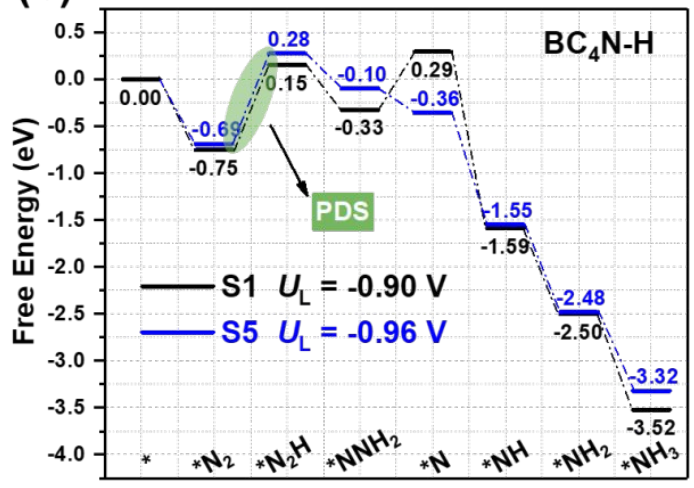

Figure S11. Free energy profiles for $\mathrm{N}_{2}$ reduction on (a) $\mathrm{BC}_{6} \mathrm{~N}_{2}-\mathrm{H}$ and (b) $\mathrm{BC}_{4} \mathrm{~N}-\mathrm{H}$ via distal pathways.

Table S7. The calculated data for thermodynamic quantities of intermediates at $\mathrm{BC}_{6} \mathrm{~N}_{2}-$ $\mathrm{H}: \mathrm{S} 1$ site along the distal pathway. $\Delta G_{\mathrm{PDS}}$ is in bold, and $G_{\mathrm{r}}$ is the free energy relative to the $*+\mathrm{N}_{2}(\mathrm{~g})$ state.

\begin{tabular}{ccccccc}
\hline \hline \multicolumn{3}{c}{ Distal@ $@ \mathrm{BC}_{6} \mathrm{~N}_{2}-\mathrm{H}: \mathrm{S} 1$} & \multicolumn{2}{c}{$\Delta E(* \mathrm{~N} 2)=-1.71 \mathrm{eV}$} & \multicolumn{2}{c}{$U_{\mathrm{L}}=-0.61 \mathrm{~V}$} \\
\hline Step & State & $E_{\mathrm{t}}-E_{*}(\mathrm{eV})$ & $\mathrm{ZPE}(\mathrm{eV})$ & $G(\mathrm{eV})$ & $\Delta G(\mathrm{eV})$ & $G_{\mathrm{r}}(\mathrm{eV})$ \\
\hline 0 & $* \mathrm{~N}_{2}$ & -18.35 & 0.24 & -18.11 & -1.04 & -1.04 \\
1 & $* \mathrm{~N}_{2} \mathrm{H}$ & -21.43 & 0.51 & -20.92 & 0.61 & -0.43 \\
2 & $* \mathrm{NNH}_{2}$ & -25.50 & 0.86 & -24.64 & -0.29 & -0.72 \\
3 & $* \mathrm{~N}$ & -8.61 & 0.12 & -8.50 & 0.36 & -0.36 \\
4 & $* \mathrm{NH}$ & -14.07 & 0.45 & -13.62 & -1.70 & -2.06 \\
5 & $* \mathrm{NH}_{2}$ & -19.09 & 0.76 & -18.33 & -1.28 & -3.34 \\
6 & $* \mathrm{NH}_{3}$ & -23.20 & 1.07 & -22.13 & -0.38 & -3.72 \\
\hline \hline
\end{tabular}

Table S8. The calculated data for thermodynamic quantities of intermediates at $\mathrm{BC}_{6} \mathrm{~N}_{2}-$ $\mathrm{H}: \mathrm{S} 1$ site along the alternating pathway.

\begin{tabular}{ccccccc}
\hline \hline \multicolumn{3}{c}{ Alternating@ $@ \mathrm{BC}_{6} \mathrm{~N}_{2}-\mathrm{H}: \mathrm{S} 1$} & \multicolumn{2}{c}{$\Delta E\left(* \mathrm{~N}_{2}\right)=-1.71 \mathrm{eV}$} & \multicolumn{2}{c}{$U_{\mathrm{L}}=-0.61 \mathrm{~V}$} \\
\hline Step & State & $E_{\mathrm{t}}-E *(\mathrm{eV})$ & $\mathrm{ZPE}(\mathrm{eV})$ & $G(\mathrm{eV})$ & $\Delta G(\mathrm{eV})$ & $G_{\mathrm{r}}(\mathrm{eV})$ \\
\hline 0 & $* \mathrm{~N}_{2}$ & -18.35 & 0.24 & -18.11 & -1.04 & -1.04 \\
1 & $* \mathrm{~N}_{2} \mathrm{H}$ & -21.43 & 0.51 & -20.92 & $\mathbf{0 . 6 1}$ & -0.43 \\
2 & $* \mathrm{NHNH}$ & -25.44 & 0.84 & -24.60 & -0.25 & -0.68 \\
3 & $* \mathrm{NHNH}_{2}$ & -30.15 & 1.20 & -28.95 & -0.93 & -1.61 \\
4 & $* \mathrm{NH}_{2} \mathrm{NH}_{2}$ & -34.09 & 1.54 & -32.56 & -0.18 & -1.79 \\
5 & $* \mathrm{NH}_{2}$ & -19.09 & 0.76 & -18.33 & -1.55 & -3.34 \\
6 & $* \mathrm{NH}_{3}$ & -23.20 & 1.07 & -22.13 & -0.38 & -3.72 \\
\hline \hline
\end{tabular}


Table S9. The calculated data for thermodynamic quantities of intermediates at $\mathrm{BC}_{6} \mathrm{~N}_{2}-$ $\mathrm{H}: \mathrm{S} 1$ site along the enzymatic pathway.

\begin{tabular}{ccccccc}
\hline \hline & \multicolumn{2}{c}{ Enzymatic@ $@ \mathrm{BC}_{6} \mathrm{~N}_{2}-\mathrm{H}: \mathrm{S} 1$} & \multicolumn{2}{c}{$\Delta E\left(* * \mathrm{~N}_{2}\right)$} & $=-0.59 \mathrm{eV}$ & \multicolumn{2}{c}{$U_{\mathrm{L}}=-0.15 \mathrm{~V}$} \\
\hline Step & State & $E_{\mathrm{t}}-E *(\mathrm{eV})$ & $\mathrm{ZPE}(\mathrm{eV})$ & $G(\mathrm{eV})$ & $\Delta G(\mathrm{eV})$ & $G_{\mathrm{r}}(\mathrm{eV})$ \\
\hline 0 & $* * \mathrm{~N}_{2}$ & -17.22 & 0.21 & -17.02 & 0.06 & 0.06 \\
1 & $* * \mathrm{NNH}$ & -20.89 & 0.52 & -20.36 & $\mathbf{0 . 0 7}$ & 0.13 \\
2 & $* * \mathrm{NHNH}$ & -24.57 & 0.83 & -23.74 & 0.05 & 0.18 \\
3 & $* * \mathrm{NHNH}_{2}$ & -29.92 & 1.20 & -28.72 & -1.55 & -1.37 \\
4 & $* * \mathrm{NH}_{2} \mathrm{NH}_{2}$ & -33.87 & 1.51 & -32.36 & -0.22 & -1.59 \\
5 & $* \mathrm{NH}_{2}$ & -19.09 & 0.76 & -18.33 & -1.75 & -3.34 \\
6 & $* \mathrm{NH}_{3}$ & -23.20 & 1.07 & -22.13 & -0.38 & -3.72 \\
\hline \hline
\end{tabular}

Table S10. The calculated data for thermodynamic quantities of intermediates at $\mathrm{BC}_{6} \mathrm{~N}_{2}-\mathrm{H}: \mathrm{S} 2$ site along the distal pathway.

\begin{tabular}{ccccccc}
\hline \hline & \multicolumn{2}{c}{ Distal@ $\mathrm{BC}_{6} \mathrm{~N}_{2}-\mathrm{H}: \mathrm{S} 2$} & \multicolumn{2}{c}{$\Delta E\left(* \mathrm{~N}_{2}\right)=-1.38 \mathrm{eV}$} & \multicolumn{2}{c}{$U_{\mathrm{L}}=-0.34 \mathrm{~V}$} \\
\hline Step & State & $E_{\mathrm{t}}-E *(\mathrm{eV})$ & $\mathrm{ZPE}(\mathrm{eV})$ & $G(\mathrm{eV})$ & $\Delta G(\mathrm{eV})$ & $G_{\mathrm{r}}(\mathrm{eV})$ \\
\hline 0 & $* \mathrm{~N}_{2}$ & -18.02 & 0.23 & -17.79 & -0.72 & -0.72 \\
1 & $* \mathrm{NNH}$ & -21.38 & 0.51 & -20.88 & $\mathbf{0 . 3 4}$ & -0.38 \\
2 & $* \mathrm{NNH}_{2}$ & -25.07 & 0.84 & -24.23 & 0.07 & -0.31 \\
3 & $* \mathrm{~N}$ & -8.31 & 0.11 & -8.20 & 0.24 & -0.07 \\
4 & $* \mathrm{NH}$ & -13.49 & 0.37 & -13.13 & -1.50 & -1.57 \\
5 & $* \mathrm{NH}_{2}$ & -19.07 & 0.77 & -18.31 & -1.75 & -3.32 \\
6 & $* \mathrm{NH}_{3}$ & -22.76 & 1.06 & -21.70 & 0.03 & -3.30 \\
\hline \hline
\end{tabular}

Table S11. The calculated data for thermodynamic quantities of intermediates at $\mathrm{BC}_{6} \mathrm{~N}_{2}-\mathrm{H}: \mathrm{S} 2$ site along the alternating pathway.

\begin{tabular}{ccccccc}
\hline \hline \multicolumn{3}{c}{ Alternating@ $\mathrm{BC}_{6} \mathrm{~N}_{2}-\mathrm{H}: \mathrm{S} 2$} & \multicolumn{2}{c}{$\Delta E\left(* \mathrm{~N}_{2}\right)=-1.38 \mathrm{eV}$} & \multicolumn{2}{c}{$U_{\mathrm{L}}=-0.34 \mathrm{~V}$} \\
\hline Step & State & $E_{\mathrm{t}}-E *(\mathrm{eV})$ & $\mathrm{ZPE}(\mathrm{eV})$ & $G(\mathrm{eV})$ & $\Delta G(\mathrm{eV})$ & $G_{\mathrm{r}}(\mathrm{eV})$ \\
\hline 0 & $* \mathrm{~N}_{2}$ & -18.02 & 0.23 & -17.79 & -0.72 & -0.72 \\
1 & $* \mathrm{NNH}$ & -21.38 & 0.51 & -20.88 & $\mathbf{0 . 3 4}$ & -0.38 \\
2 & $* \mathrm{NHNH}$ & -25.13 & 0.86 & -24.27 & 0.03 & -0.35 \\
3 & $* \mathrm{NHNH}_{2}$ & -29.96 & 1.20 & -28.76 & -1.07 & -1.42 \\
4 & $* \mathrm{NH}_{2} \mathrm{NH}_{2}$ & -33.69 & 1.52 & -32.17 & 0.01 & -1.41 \\
5 & $* \mathrm{NH}_{2}$ & -19.07 & 0.77 & -18.31 & -1.92 & -3.33 \\
6 & $* \mathrm{NH}_{3}$ & -22.76 & 1.06 & -21.70 & 0.03 & -3.30 \\
\hline \hline
\end{tabular}


Table S12. The calculated data for thermodynamic quantities of intermediates at $\mathrm{BC}_{6} \mathrm{~N}_{2}-\mathrm{H}: \mathrm{S} 2$ site along the enzymatic pathway.

\begin{tabular}{ccccccc}
\hline \hline & \multirow{2}{*}{ Enzymatic $@ \mathrm{BC}_{6} \mathrm{~N}_{2}-\mathrm{H}: \mathrm{S} 2$} & \multicolumn{2}{c}{$\Delta E\left(* * \mathrm{~N}_{2}\right)$} & $=-0.16 \mathrm{eV}$ & \multicolumn{2}{c}{$U_{\mathrm{L}}=-0.27 \mathrm{~V}$} \\
\hline Step & State & $E_{\mathrm{t}}-E *(\mathrm{eV})$ & $\mathrm{ZPE}(\mathrm{eV})$ & $G(\mathrm{eV})$ & $\Delta G(\mathrm{eV})$ & $G_{\mathrm{r}}(\mathrm{eV})$ \\
\hline 0 & $* * \mathrm{~N}_{2}$ & -16.80 & 0.20 & -16.60 & 0.47 & 0.47 \\
1 & $* * \mathrm{NNH}$ & -20.77 & 0.53 & -20.25 & -0.22 & 0.25 \\
2 & $* * \mathrm{NHNH}$ & -24.22 & 0.82 & -23.40 & $\mathbf{0 . 2 7}$ & 0.52 \\
3 & $* * \mathrm{NHNH}_{2}$ & -29.84 & 1.21 & -28.64 & -1.82 & -1.30 \\
4 & $* * \mathrm{NH}_{2} \mathrm{NH}_{2}$ & -33.64 & 1.50 & -32.14 & -0.08 & -1.38 \\
5 & $* \mathrm{NH}_{2}$ & -19.07 & 0.77 & -18.31 & -1.95 & -3.33 \\
6 & $* \mathrm{NH}_{3}$ & -22.76 & 1.06 & -21.70 & 0.03 & -3.30 \\
\hline \hline
\end{tabular}

Table S13. The calculated data for thermodynamic quantities of intermediates at $\mathrm{BC}_{6} \mathrm{~N}_{2}-\mathrm{H}: \mathrm{S} 5$ site along the distal pathway.

\begin{tabular}{ccccccc}
\hline \hline & \multicolumn{2}{c}{ Distal@ $\mathrm{BC}_{6} \mathrm{~N}_{2}-\mathrm{H}: \mathrm{S} 5$} & \multicolumn{2}{c}{$\Delta E\left(* \mathrm{~N}_{2}\right)=-1.73 \mathrm{eV}$} & \multicolumn{2}{c}{$U_{\mathrm{L}}=-0.75 \mathrm{~V}$} \\
\hline Step & State & $E_{\mathrm{t}}-E *(\mathrm{eV})$ & $\mathrm{ZPE}(\mathrm{eV})$ & $G(\mathrm{eV})$ & $\Delta G(\mathrm{eV})$ & $G_{\mathrm{r}}(\mathrm{eV})$ \\
\hline 0 & $* \mathrm{~N}_{2}$ & -18.36 & 0.24 & -18.12 & -1.05 & -1.05 \\
1 & $* \mathrm{NNH}$ & -21.30 & 0.51 & -20.79 & $\mathbf{0 . 7 5}$ & -0.30 \\
2 & $* \mathrm{NNH}_{2}$ & -25.37 & 0.85 & -24.52 & -0.30 & -0.60 \\
3 & $* \mathrm{~N}$ & -8.65 & 0.12 & -8.53 & 0.21 & -0.39 \\
4 & $* \mathrm{NH}$ & -12.68 & 0.37 & -12.31 & -0.36 & -0.75 \\
5 & $* \mathrm{NH}_{2}$ & -19.07 & 0.76 & -18.31 & -2.57 & -3.32 \\
6 & $* \mathrm{NH}_{3}$ & -23.09 & 1.07 & -22.02 & -0.29 & -3.62 \\
\hline \hline
\end{tabular}

Table S14. The calculated data for thermodynamic quantities of intermediates at $\mathrm{BC}_{6} \mathrm{~N}_{2}-\mathrm{H}: \mathrm{S} 5$ site along the alternating pathway.

\begin{tabular}{ccccccc}
\hline \hline \multicolumn{3}{c}{ Alternating@ $\mathrm{BC}_{6} \mathrm{~N}_{2}-\mathrm{H}: \mathrm{S} 5$} & \multicolumn{2}{c}{$\Delta E\left(* \mathrm{~N}_{2}\right)=-1.73 \mathrm{eV}$} & \multicolumn{2}{c}{$U_{\mathrm{L}}=-0.75 \mathrm{~V}$} \\
\hline Step & State & $E_{\mathrm{t}}-E *(\mathrm{eV})$ & $\mathrm{ZPE}(\mathrm{eV})$ & $G(\mathrm{eV})$ & $\Delta G(\mathrm{eV})$ & $G_{\mathrm{r}}(\mathrm{eV})$ \\
\hline 0 & $* \mathrm{~N}_{2}$ & -18.36 & 0.24 & -18.12 & -1.05 & -1.05 \\
1 & $* \mathrm{NNH}$ & -21.30 & 0.51 & -20.79 & $\mathbf{0 . 7 5}$ & -0.30 \\
2 & $* \mathrm{NHNH}$ & -25.43 & 0.87 & -24.55 & -0.34 & -0.64 \\
3 & $* \mathrm{NHNH}_{2}$ & -30.08 & 1.19 & -28.89 & -0.91 & -1.55 \\
4 & $* \mathrm{NH}_{2} \mathrm{NH}_{2}$ & -34.03 & 1.53 & -32.50 & -0.19 & -1.74 \\
5 & $* \mathrm{NH}_{2}$ & -19.07 & 0.76 & -18.31 & -1.59 & -3.33 \\
6 & $* \mathrm{NH}_{3}$ & -23.09 & 1.07 & -22.02 & -0.29 & -3.62 \\
\hline \hline
\end{tabular}


Table S15. The calculated data for thermodynamic quantities of intermediates at $\mathrm{BC}_{6} \mathrm{~N}_{2}-\mathrm{H}: \mathrm{S} 5$ site along the enzymatic pathway.

\begin{tabular}{ccccccc}
\hline \hline \multicolumn{3}{c}{ Enzymatic $@ \mathrm{BC}_{6} \mathrm{~N}_{2}-\mathrm{H}: \mathrm{S} 5$} & \multicolumn{2}{c}{$\Delta E\left(* * \mathrm{~N}_{2}\right)=-0.53 \mathrm{eV}$} & \multicolumn{2}{c}{$U_{\mathrm{L}}=-0.17 \mathrm{~V}$} \\
\hline Step & State & $E_{\mathrm{t}}-E *(\mathrm{eV})$ & $\mathrm{ZPE}(\mathrm{eV})$ & $G(\mathrm{eV})$ & $\Delta G(\mathrm{eV})$ & $G_{\mathrm{r}}(\mathrm{eV})$ \\
\hline 0 & $* * \mathrm{~N}_{2}$ & -17.17 & 0.20 & -16.97 & 0.10 & 0.10 \\
1 & $* * \mathrm{NNH}$ & -20.75 & 0.53 & -20.22 & $\mathbf{0 . 1 7}$ & 0.27 \\
2 & $* * \mathrm{NHNH}$ & -24.50 & 0.83 & -23.67 & -0.03 & 0.24 \\
3 & $* * \mathrm{NHNH}_{2}$ & -29.90 & 1.20 & -28.70 & -1.61 & -1.37 \\
4 & $* * \mathrm{NH}_{2} \mathrm{NH}_{2}$ & -33.72 & 1.51 & -32.20 & -0.07 & -1.44 \\
5 & $* \mathrm{NH}_{2}$ & -19.07 & 0.76 & -18.31 & -1.89 & -3.33 \\
6 & $* \mathrm{NH}_{3}$ & -23.09 & 1.07 & -22.02 & -0.29 & -3.62 \\
\hline \hline
\end{tabular}

Table S16. The calculated data for thermodynamic quantities of intermediates at $\mathrm{BC}_{4} \mathrm{~N}-$ $\mathrm{H}: \mathrm{S} 1$ site along the distal pathway.

\begin{tabular}{ccccccc}
\hline \hline & \multicolumn{2}{c}{ Distal@BC $\mathrm{BC}_{4} \mathrm{~N}-\mathrm{H}: \mathrm{S} 1$} & \multicolumn{2}{c}{$\Delta E\left(* \mathrm{~N}_{2}\right)=-1.41 \mathrm{eV}$} & \multicolumn{2}{c}{$U_{\mathrm{L}}=-0.90 \mathrm{~V}$} \\
\hline Step & State & $E_{\mathrm{t}}-E_{*}(\mathrm{eV})$ & $\mathrm{ZPE}(\mathrm{eV})$ & $G(\mathrm{eV})$ & $\Delta G(\mathrm{eV})$ & $G_{\mathrm{r}}(\mathrm{eV})$ \\
\hline 0 & $* \mathrm{~N}_{2}$ & -18.05 & 0.23 & -17.82 & -0.75 & -0.75 \\
1 & $* \mathrm{~N}_{2} \mathrm{H}$ & -20.80 & 0.46 & -20.34 & $\mathbf{0 . 9 0}$ & 0.15 \\
2 & $* \mathrm{NNH}_{2}$ & -25.06 & 0.82 & -24.24 & -0.48 & -0.33 \\
3 & $* \mathrm{~N}$ & -7.95 & 0.11 & -7.84 & 0.62 & 0.29 \\
4 & $* \mathrm{NH}$ & -13.59 & 0.44 & -13.15 & -1.88 & -1.59 \\
5 & $* \mathrm{NH}_{2}$ & -18.23 & 0.74 & -17.49 & -0.91 & -2.50 \\
6 & $* \mathrm{NH}_{3}$ & -22.99 & 1.07 & -21.93 & -1.02 & -3.52 \\
\hline \hline
\end{tabular}

Table S17. The calculated data for thermodynamic quantities of intermediates at $\mathrm{BC}_{4} \mathrm{~N}-$ $\mathrm{H}: \mathrm{S} 1$ site along the alternating pathway.

\begin{tabular}{ccccccc}
\hline \hline & \multicolumn{2}{c}{ Alternating $@ \mathrm{BC}_{4} \mathrm{~N}-\mathrm{H}: \mathrm{S} 1$} & \multicolumn{2}{c}{$\Delta E\left(* \mathrm{~N}_{2}\right)=-1.41 \mathrm{eV}$} & \multicolumn{2}{c}{$U_{\mathrm{L}}=-0.90 \mathrm{~V}$} \\
\hline Step & State & $E_{\mathrm{t}}-E_{*}(\mathrm{eV})$ & $\mathrm{ZPE}(\mathrm{eV})$ & $G(\mathrm{eV})$ & $\Delta G(\mathrm{eV})$ & $G_{\mathrm{r}}(\mathrm{eV})$ \\
\hline 0 & $* \mathrm{~N}_{2}$ & -18.05 & 0.23 & -17.82 & -0.75 & -0.75 \\
1 & $* \mathrm{NNH}$ & -20.80 & 0.46 & -20.34 & $\mathbf{0 . 9 0}$ & 0.15 \\
2 & $* \mathrm{NHNH}$ & -25.34 & 0.84 & -24.49 & -0.73 & -0.58 \\
3 & $* \mathrm{NHNH}_{2}$ & -29.72 & 1.20 & -28.52 & -0.60 & -1.18 \\
4 & $* \mathrm{NH}_{2} \mathrm{NH}_{2}$ & -33.94 & 1.53 & -32.41 & -0.47 & -1.65 \\
5 & $* \mathrm{NH}_{2}$ & -18.23 & 0.74 & -17.49 & -0.86 & -2.51 \\
6 & $* \mathrm{NH}_{3}$ & -22.99 & 1.07 & -21.93 & -1.02 & -3.52 \\
\hline \hline
\end{tabular}


Table S18. The calculated data for thermodynamic quantities of intermediates at $\mathrm{BC}_{4} \mathrm{~N}-$ $\mathrm{H}: \mathrm{S} 1$ site along the enzymatic pathway.

\begin{tabular}{ccccccc}
\hline \hline & \multicolumn{2}{c}{ Enzymatic@BC $\mathrm{BC}_{4} \mathrm{~N}-\mathrm{H}: \mathrm{S} 1$} & \multicolumn{2}{c}{$\Delta E\left(* \mathrm{~N}_{2}\right)=-0.11 \mathrm{eV}$} & \multicolumn{2}{c}{$U_{\mathrm{L}}=-0.39 \mathrm{~V}$} \\
\hline Step & State & $E_{\mathrm{t}}-E_{*}(\mathrm{eV})$ & $\mathrm{ZPE}(\mathrm{eV})$ & $G(\mathrm{eV})$ & $\Delta G(\mathrm{eV})$ & $G_{\mathrm{r}}(\mathrm{eV})$ \\
\hline 0 & $* * \mathrm{~N}_{2}$ & -16.74 & 0.19 & -16.55 & 0.52 & 0.52 \\
1 & $* * \mathrm{NNH}$ & -20.08 & 0.49 & -19.59 & $\mathbf{0 . 3 9}$ & 0.91 \\
2 & $* * \mathrm{NHNH}$ & -24.04 & 0.83 & -23.21 & -0.20 & 0.71 \\
3 & $* * \mathrm{NHNH}_{2}$ & -29.12 & 1.17 & -27.95 & -1.32 & -0.61 \\
4 & $* * \mathrm{NH}_{2} \mathrm{NH}_{2}$ & -33.72 & 1.52 & -32.20 & -0.82 & -1.43 \\
5 & $* \mathrm{NH}_{2}$ & -18.23 & 0.74 & -17.49 & -1.07 & -2.50 \\
6 & $* \mathrm{NH}_{3}$ & -22.99 & 1.07 & -21.93 & -1.02 & -3.52 \\
\hline \hline
\end{tabular}

Table S19. The calculated data for thermodynamic quantities of intermediates at $\mathrm{BC}_{4} \mathrm{~N}-$ $\mathrm{H}$ :S5 site along the distal pathway.

\begin{tabular}{ccccccc}
\hline \hline & \multicolumn{2}{c}{ Distal@BC ${ }_{4} \mathrm{~N}-\mathrm{H}: \mathrm{S} 5$} & \multicolumn{2}{c}{$\Delta E\left(* \mathrm{~N}_{2}\right)=-1.35 \mathrm{eV}$} & \multicolumn{2}{c}{$U_{\mathrm{L}}=-0.96 \mathrm{~V}$} \\
\hline Step & State & $E_{\mathrm{t}}-E_{*}(\mathrm{eV})$ & $\mathrm{ZPE}(\mathrm{eV})$ & $G(\mathrm{eV})$ & $\Delta G(\mathrm{eV})$ & $G_{\mathrm{r}}(\mathrm{eV})$ \\
\hline 0 & $* \mathrm{~N}_{2}$ & -17.98 & 0.23 & -17.76 & -0.69 & -0.69 \\
1 & $* \mathrm{~N}_{2} \mathrm{H}$ & -20.71 & 0.49 & -20.22 & $\mathbf{0 . 9 6}$ & 0.27 \\
2 & $* \mathrm{NNH}_{2}$ & -24.87 & 0.85 & -24.01 & -0.37 & -0.10 \\
3 & $* \mathrm{~N}$ & -8.60 & 0.11 & -8.49 & -0.26 & -0.36 \\
4 & $* \mathrm{NH}$ & -13.54 & 0.43 & -13.10 & -1.19 & -1.55 \\
5 & $* \mathrm{NH}_{2}$ & -18.19 & 0.74 & -17.45 & -0.93 & -2.48 \\
6 & $* \mathrm{NH}_{3}$ & -22.78 & 1.05 & -21.73 & -0.85 & -3.32 \\
\hline \hline
\end{tabular}

Table S20. The calculated data for thermodynamic quantities of intermediates at $\mathrm{BC}_{4} \mathrm{~N}-$ $\mathrm{H}: \mathrm{S} 5$ site along the alternating pathway.

\begin{tabular}{ccccccc}
\hline \hline & \multicolumn{2}{c}{ Alternating $@ \mathrm{BC}_{4} \mathrm{~N}-\mathrm{H}: \mathrm{S} 5$} & \multicolumn{2}{c}{$\Delta E\left(* \mathrm{~N}_{2}\right)=-1.35 \mathrm{eV}$} & \multicolumn{2}{c}{$U_{\mathrm{L}}=-0.96 \mathrm{~V}$} \\
\hline Step & State & $E_{\mathrm{t}}-E_{*}(\mathrm{eV})$ & $\mathrm{ZPE}(\mathrm{eV})$ & $G(\mathrm{eV})$ & $\Delta G(\mathrm{eV})$ & $G_{\mathrm{r}}(\mathrm{eV})$ \\
\hline 0 & $* \mathrm{~N}_{2}$ & -17.98 & 0.23 & -17.76 & -0.69 & -0.69 \\
1 & $* \mathrm{NNH}$ & -20.71 & 0.49 & -20.22 & $\mathbf{0 . 9 6}$ & 0.27 \\
2 & $* \mathrm{NHNH}$ & -25.25 & 0.84 & -24.41 & -0.77 & -0.50 \\
3 & $* \mathrm{NHNH}_{2}$ & -29.77 & 1.20 & -28.57 & -0.73 & -1.23 \\
4 & $* \mathrm{NH}_{2} \mathrm{NH}_{2}$ & -33.84 & 1.48 & -32.36 & -0.37 & -1.60 \\
5 & $* \mathrm{NH}_{2}$ & -18.19 & 0.74 & -17.45 & -0.87 & -2.47 \\
6 & $* \mathrm{NH}_{3}$ & -22.78 & 1.05 & -21.73 & -0.85 & -3.32 \\
\hline \hline
\end{tabular}


Table S21. The calculated data for thermodynamic quantities of intermediates at $\mathrm{BC}_{4} \mathrm{~N}-$ $\mathrm{H}: \mathrm{S} 5$ site along the enzymatic pathway.

\begin{tabular}{ccccccc}
\hline \hline \multicolumn{2}{c}{ Enzymatic@BC $\mathrm{BC}_{4} \mathrm{~N}-\mathrm{H}: \mathrm{S} 5$} & \multicolumn{2}{c}{$\Delta E\left(* \mathrm{~N}_{2}\right)=-0.10 \mathrm{eV}$} & \multicolumn{2}{c}{$U_{\mathrm{L}}=-0.59 \mathrm{~V}$} \\
\hline Step & State & $E_{\mathrm{t}}-E_{*}(\mathrm{eV})$ & $\mathrm{ZPE}(\mathrm{eV})$ & $G(\mathrm{eV})$ & $\Delta G(\mathrm{eV})$ & $G_{\mathrm{r}}(\mathrm{eV})$ \\
\hline 0 & $* * \mathrm{~N}_{2}$ & -16.74 & 0.19 & -16.55 & 0.52 & 0.52 \\
1 & $* * \mathrm{NNH}$ & -19.89 & 0.51 & -19.38 & $\mathbf{0 . 5 9}$ & 1.11 \\
2 & $* * \mathrm{NHNH}$ & -23.90 & 0.82 & -23.08 & -0.28 & 0.83 \\
3 & $* * \mathrm{NHNH}_{2}$ & -29.07 & 1.17 & -27.90 & -1.39 & -0.56 \\
4 & $* * \mathrm{NH}_{2} \mathrm{NH}_{2}$ & -32.89 & 1.37 & -31.52 & -0.20 & -0.76 \\
5 & $* \mathrm{NH}_{2}$ & -18.19 & 0.74 & -17.45 & -1.71 & -2.47 \\
6 & $* \mathrm{NH}_{3}$ & -22.78 & 1.05 & -21.73 & -0.85 & -3.32 \\
\hline \hline
\end{tabular}

Table S22. The calculated data for thermodynamic quantities of the absorbed $\mathrm{H}^{+} / \mathrm{e}^{-}$ between each NRR intermediates at $\mathrm{BC}_{6} \mathrm{~N}_{2}-\mathrm{H}: \mathrm{S} 1$ site via the enzymatic mechanism (Table S9). The free energy change $(\Delta G)$ here, for example, for the step " $0-1$ " ( $* * \mathrm{~N}_{2}+$ $\left.\mathrm{H}^{+} / \mathrm{e}^{-} \rightarrow * * \mathrm{~N}_{2}+* \mathrm{H}\right)$, is expressed as $\Delta G=G\left(* * \mathrm{~N}_{2}+* \mathrm{H}\right)-G\left(* * \mathrm{~N}_{2}\right)-1 / 2 G\left(\mathrm{H}_{2}\right)$.

\begin{tabular}{cccccc}
\hline \hline & Enzymatic $@ \mathrm{BC}_{6} \mathrm{~N}_{2}-\mathrm{H}: \mathrm{S} 1$ & \multicolumn{2}{c}{$\boldsymbol{U}_{\mathbf{L}}=-\mathbf{0 . 1 5} \mathbf{~ V}$} \\
\hline Step & State & $E_{\mathrm{t}}-E_{*}(\mathrm{eV})$ & $\mathrm{ZPE}(\mathrm{eV})$ & $G(\mathrm{eV})$ & $\Delta G(\mathrm{eV})$ \\
\hline $0-1$ & $* * \mathrm{~N}_{2}+* \mathrm{H}$ & -20.68 & 0.44 & -20.23 & $\mathbf{0 . 2 0}$ \\
$1-2$ & $* * \mathrm{~N}_{2} \mathrm{H}+* \mathrm{H}$ & -24.41 & 0.76 & -23.66 & 0.13 \\
$2-3$ & $* * \mathrm{NHNH}+* \mathrm{H}$ & -28.18 & 1.07 & -27.11 & 0.06 \\
$3-4$ & $* * \mathrm{NHNH}_{2}+* \mathrm{H}$ & -33.41 & 1.43 & -31.98 & 0.16 \\
$4-5$ & $* * \mathrm{NH}_{2} \mathrm{NH}_{2}+* \mathrm{H}$ & -37.40 & 1.76 & -35.64 & 0.14 \\
$5-6$ & $* \mathrm{NH}_{2}+* \mathrm{H}$ & -22.61 & 1.00 & -21.61 & 0.14 \\
\hline \hline
\end{tabular}

Table S23 Summary for potential-determining steps (PDS) of B-doped carbon materials for $\mathrm{N}_{2}$ reduction. \# B substitute $\mathrm{C}$ atom; * $\mathrm{B}$ absorbed on $\mathrm{C}$ atom.

\begin{tabular}{cccc}
\hline Catalysts & PDS & $U_{\mathrm{L}}(\mathrm{V})$ & Reference \\
\hline $\mathrm{BC}_{6} \mathrm{~N}_{2}$ & $* * \mathrm{~N}_{2}+\mathrm{H} \rightarrow * * \mathrm{~N}_{2} \mathrm{H}$ & -0.47 & This work \\
$\mathrm{BC}_{4} \mathrm{~N}$ & $* * \mathrm{~N}_{2}+\mathrm{H} \rightarrow * * \mathrm{~N}_{2} \mathrm{H}$ & -0.87 & This work \\
$\mathrm{BC}_{6} \mathrm{~N}_{2}-\mathrm{H}$ & $* * \mathrm{~N}_{2}+\mathrm{H} \rightarrow * * \mathrm{~N}_{2} \mathrm{H}$ & -0.15 & This work \\
BC4N-H & $* * \mathrm{~N}_{2}+\mathrm{H} \rightarrow * * \mathrm{~N}_{2} \mathrm{H}$ & -0.39 & This work \\
B@g-C $\mathrm{C}_{4}$ & $* \mathrm{NH}_{2}-* \mathrm{NH}_{2}+\mathrm{H} \rightarrow * \mathrm{NH}_{2} * \mathrm{NH}_{3}$ & -0.20 & Refs. 18 \\
B@ $\mathrm{C}_{2} \mathrm{~N}$ & $* * \mathrm{~N}_{2} \mathrm{H}+\mathrm{H} \rightarrow * * \mathrm{~N}_{2} \mathrm{H}_{2}$ & -0.15 & Refs. 17 \\
B@graphene\# & $* \mathrm{NH}_{2}+\mathrm{H} \rightarrow * \mathrm{NH}_{3}$ & -0.43 & Joule 2018, 2 (8), \\
B@graphene* & $* \mathrm{~N}+\mathrm{H} \rightarrow * \mathrm{NH}^{*}$ & -0.31 & Refs. 20 \\
B@g-CN & $* * \mathrm{~N}_{2}+\mathrm{H} \rightarrow * * \mathrm{~N}_{2} \mathrm{H}$ & -0.31 & Refs. 21 \\
$\mathrm{B}_{1} @ \mathrm{C}_{2} \mathrm{~N}$ & $* * \mathrm{~N}_{2}+\mathrm{H} \rightarrow * * \mathrm{~N}_{2} \mathrm{H}$ & -0.45 & Refs. 23 \\
$\mathrm{B}_{2} @ \mathrm{C}_{2} \mathrm{~N}$ & $* * \mathrm{~N}_{2}+\mathrm{H} \rightarrow * * \mathrm{~N}_{2} \mathrm{H}$ & -0.35 & Refs. 23 \\
\hline
\end{tabular}



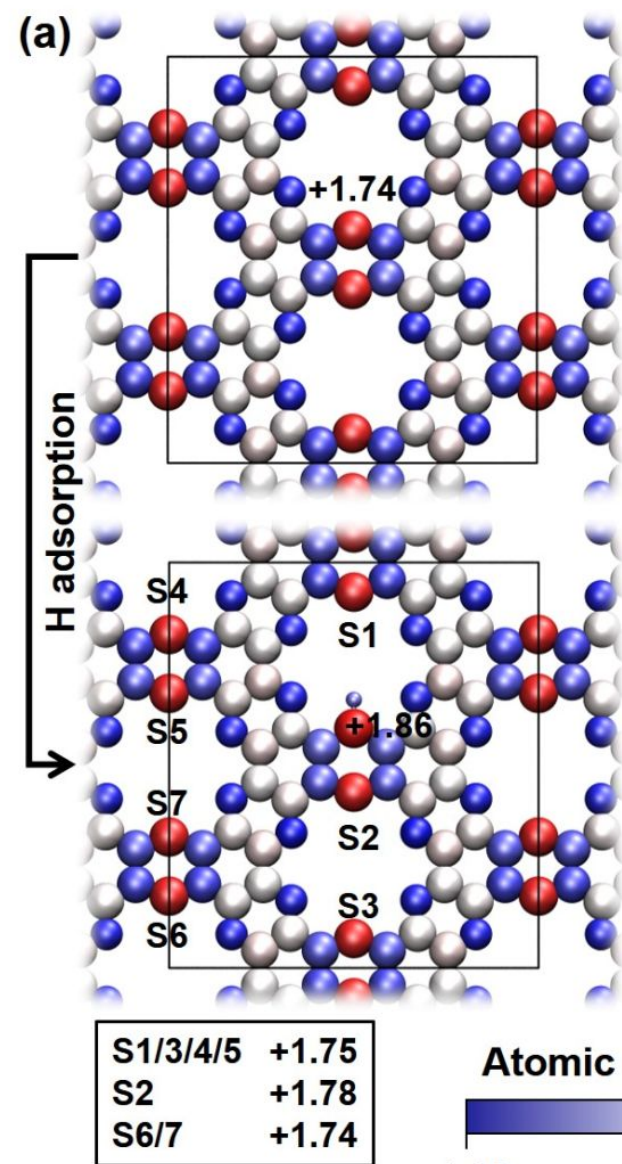

(b)

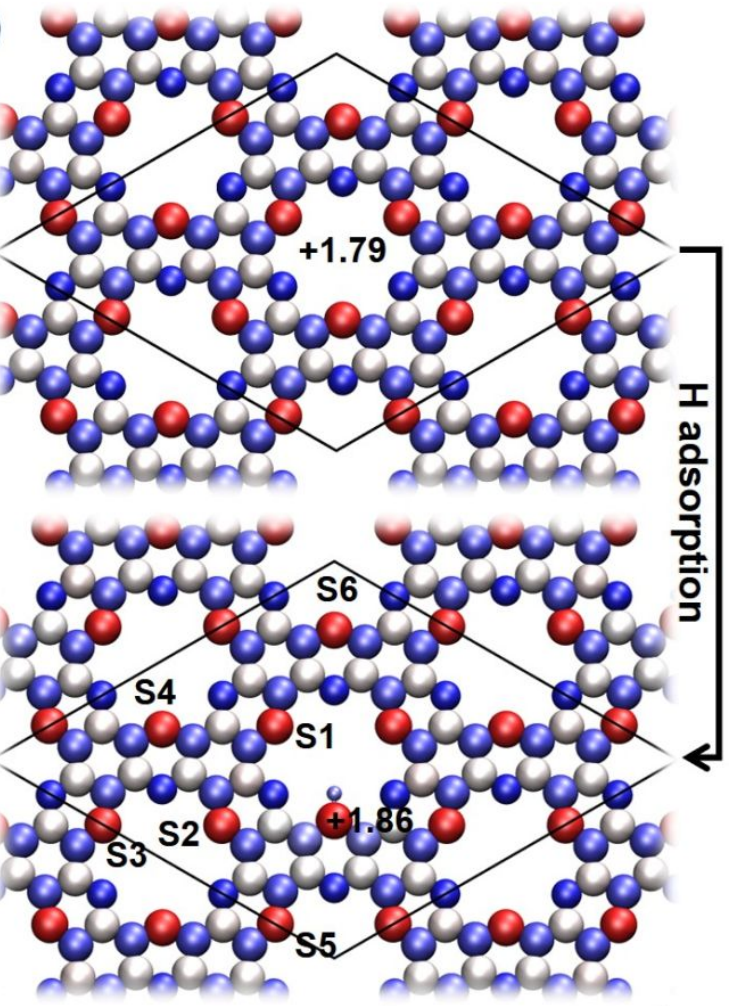

\begin{tabular}{|ll|}
\hline S1 & +1.81 \\
S2 & +1.80 \\
S3/4 & +1.78 \\
S5/6 & +1.79 \\
\hline
\end{tabular}

Figure S12. Atomic charge density of $\mathrm{B}$ atoms before and after $\mathrm{H}$ adsorption for (a) $\mathrm{BC}_{6} \mathrm{~N}_{2}$ and (b) $\mathrm{BC}_{4} \mathrm{~N}$ monolayers.
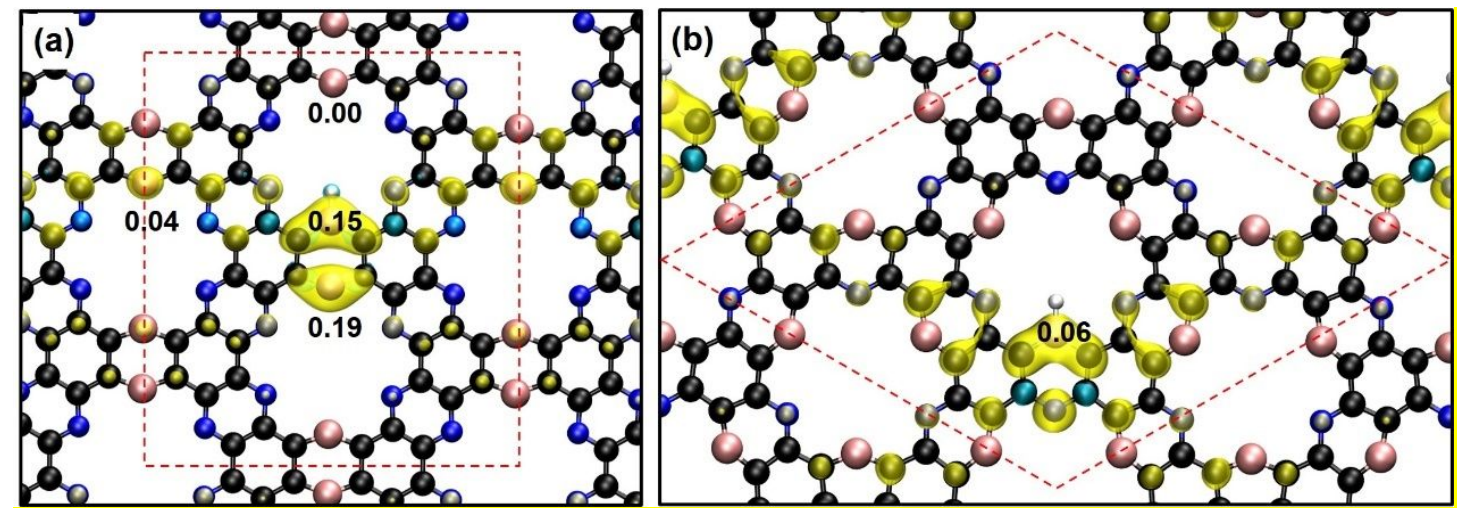

Figure S13. Magnetism (spin density) distribution for $\mathrm{BC}_{6} \mathrm{~N}_{2}-\mathrm{H} \mathrm{BC}_{4} \mathrm{~N}-\mathrm{H}$ monolayers. The isosurface value is $0.005 \mu_{\mathrm{B}} / \AA^{3}$. The yellow and cyan regions represent the spin-up and spin-down density, respectively.

\section{References}

1. Skúlason, E.; Bligaard, T.; Gudmundsdóttir, S.; Studt, F.; Rossmeisl, J.; AbildPedersen, F.; Vegge, T.; Jónsson, H.; Nørskov, J. K., A theoretical evaluation of possible transition metal electro-catalysts for N2 reduction. Physical Chemistry Chemical Physics 2012, 14 (3), 1235-1245. 
2. Montoya, J. H.; Tsai, C.; Vojvodic, A.; Nørskov, J. K., The Challenge of Electrochemical Ammonia Synthesis: A New Perspective on the Role of Nitrogen Scaling Relations. ChemSusChem 2015, 8 (13), 2180-2186.

3. III, R. D. J. NIST Computational Chemistry Comparison and Benchmark Database. http://cccbdb.nist.gov.

4. van der Ham, C. J. M.; Koper, M. T. M.; Hetterscheid, D. G. H., Challenges in reduction of dinitrogen by proton and electron transfer. Chemical Society Reviews 2014, 43 (15), 5183-5191.

5. Budyka, M. F.; Zyubina, T. S.; Ryabenko, A. G.; Lin, S. H.; Mebel, A. M., Bond lengths and diameters of armchair single wall carbon nanotubes. Chemical Physics Letters 2005, 407 (4), 266-271. 